\title{
Advanced magnetic resonance imaging techniques in brain tumours surgical planning
}

\author{
Giada Zoccatelli $^{1}$, Franco Alessandrini ${ }^{1}$, Alberto Beltramello ${ }^{1}$, Andrea Talacchi ${ }^{2}$ \\ ${ }^{1}$ Neuroradiology Unit, University Hospital of Verona, Verona, Italy \\ ${ }^{2}$ Section of Neurosurgery, Department of Neuroscience, University of Verona, Verona, Italy \\ Email: giada.zoccatelli@ospedaleuniverona.it
}

Received 14 January 2013; revised 20 February 2013; accepted 28 February 2013

\begin{abstract}
Morphological assessment using Computed Tomography (CT) or Magnetic Resonance Imaging (MRI) is still the workhorse of tumor detection and diagnosis. In particular, MRI provides detailed information about cerebral tumor anatomy, cellular metabolism and hemodynamic features, making it a fundamental tool for a correct diagnosis, treatment and monitoring of the disease. Various new functional imaging modalities assessing tissue microstructure and physiology have increased the scope of neuro imaging and raised expectations among clinicians. This article provides an overview of the most advanced MR imaging techniques (functional MRI, perfusion-weighted imaging, diffusion-weighted imaging and MR spectroscopy) now available for neurosurgical planning and their role in brain tumors assessment. Their pros and cons are analyzed in order to find out which one may be chosen as best diagnostic pre-surgical protocol. At the moment none of the single techniques can be considered the golden standard; only the integration of advanced and conventional MR imaging proves to be a reliable tool in the hands of the neuroradiologist and neurosurgeon, thus maximazing tumor resection and function preservation.
\end{abstract}

Keywords: Magnetic Resonance Imaging; Brain Tumor; Neurosurgery; Brain Mapping

\section{INTRODUCTION}

MRI is a medical diagnostic technique that creates images of the human body using the principle of nuclear magnetic resonance. It can generate thin-section images of any part of the human body-from any angle and direction. An MRI image is not a photograph; it is actually a computerized map or image of radio signals emitted by the human body. MRI is therefore superior to CT scan because CT scan uses ionizing radiation, whereas MRI uses harmless radio waves. MRI foundations date back to the year of 1946. Felix Bloch and Edward Purcell independently discovered the magnetic resonance phenomena; they found that when certain naturally occurring nuclei were placed in a magnetic field they absorbed energy in the radio frequency range of the electromagnetic spectrum. This energy was re-emitted when the nuclei relaxed back to their original state, a phenomenon termed Nuclear Magnetic Resonance (NMR). With this discovery, NMR became an important analytical method in the study of the composition of chemical compounds, that later gave birth to its associated specialized technique called NMR Spectroscopy. Felix Bloch and Edward Purcell were awarded the Nobel Prize for Physics in 1952 for this discovery. During the 1950s and 1960s NMR spectroscopy became a widely used technique for the invasive analysis of small samples and many of its applications were at the microscopic level using smallbore, high-field magnets. Thus, up until the 1970s it was being used mainly for chemical and physical analysis. The advent of computed tomography (using computer techniques to develop images from NMR information) in the early 70's has revolutionized the world of radiology, setting new standards for medical imaging giving birth to MRI (Magnetic Resonance imaging). In 1971 Raymond Damadian showed that nuclear magnetic relaxation times of tissues and tumors differed, motivating scientists to use NMR to study disease. In 1973, Paul Lauterbur described his research on the high level of contrast that could be realized with MR in clinical settings. His approach was based on the independent discoveries made by Bloch and Purcell three decades earlier. Moreover, Sir Peter Mansfield developed methodology to analyze the signals and assemble them rapidly into three-dimensional images. In 1977, the first MRI exam was performed on a human being. The procedure was long and complicated, taking over 5 hours to produce a single image. Although researchers had struggled for over 7 years to reach this point, by today's standards, the image was unrefined and coarse. Many scientists over the next 20 years developed 
MRI into the technology that we now know today. Critical advances in technology development now allow physicians to image in seconds what used to take hours. Dr. Lauterbur, a long-time NIH grantee, and Dr. Mansfield were recently awarded the 2003 Nobel Prize in Physiology or Medicine for their discoveries in MRI.

MRI has been used successfully for over 15 years to generate soft tissue images of the human body, making it the technique of choice for the routine diagnosis of many diseases and disease processes. Today more than 60 million noninvasive, diagnostic MRI procedures are performed worldwide each year. This is how and why the development of MRI has revolutionized the practice of medicine.

MRI provides excellent contrast between the different soft tissues of the body, unparalleled if compared to other medical imaging techniques such as CT. The use of ionizing radiation by a $\mathrm{CT}$ scanner to acquire images, make it a good tool for examining tissue composed of elements of a higher atomic number than the tissue surrounding them, such as bone and calcifications. Nevertheless, ionizing radiations used to produce images can damage DNA and subsequently cause cancer. There is a small increased risk of cancer with CT scans. It is estimated that $0.4 \%$ of current cancers in the United States are due to CTs performed in the past and that this may increase to as high as $1.5 \%-2 \%$ with 2007 rates of CT usage [1]. Thus, contrast in CT images is generated purely by X-ray attenuation and it may be enhanced by the use of contrast agents. Contrast agents for CT contain elements of a high atomic number relative to tissue, such as iodine or barium. MRI, on the other hand, uses nonionizing Radio Frequency (RF) signals to acquire its images and is best suited for soft tissue characterization. While CT provides good spatial resolution (the ability to distinguish two separate structures an arbitrarily small distance from each other), MRI provides comparable resolution with far better contrast resolution (the ability to distinguish the differences between two arbitrarily similar but not identical tissues). This makes it especially useful in imaging the brain, muscles, the heart, both in General as well as in Neuroradiology. The basis of this ability is the complex availability of pulse sequences that the modern medical MRI scanner includes, each of which is optimized to provide image contrast based on the chemical sensitivity of MRI. This image contrast may be eventually improved by the use of a contrast agent (Gadolinium chelate or Gd-DTPA, Diethylene Triamine Pent Acetate) that acts differently from iodinated contrast media due to its paramagnetic properties which alter tissue relaxation times. Commonly used MRI contrast agents may be contraindicated in persons with significant permanent or transient kidney dysfunction. There are several other safety issues concerning MRI use, as acer- tain number of features can give rise to risks. These include:

- Magnetic fields: generally speaking, metallic medical implants are generally considered contraindications for MRI examinations; they may be allowed under certain specific conditions. Patients are therefore always asked for complete information about all implants before entering the room for an MRI scan. Precautions are focused on pacemakers and metallic vascular implants. To reduce such risks, efforts are raised to create compatible materials; moreover, specialized protocols have been developed to permit the safe scanning of selected implants and pacing devices. Ferromagnetic foreign bodies such as shell fragments or metallic implants such as surgical prostheses and ferromagnetic aneurysm clips are also potential risks. Interaction of the magnetic and radio frequency fields with such objects can lead to trauma due to movement of the object in the magnetic field or thermal injury from radio-frequency induction heating of the object. Titanium and its alloys are safe from movement from the magnetic field. Cardiovascular stents are now considered safe, however.

- Peripheral nerve stimulation: the rapid switching on and off of the magnetic field gradients is capable of causing nerve stimulation. Although it was not a problem for the slow, weak gradients used in the early days of MRI, the strong, rapidly switched gradients used in new and latest imaging techniques sequences are indeed capable of inducing peripheral nerve stimulation. As a result American and European regulatory agencies have limited the power of MR scanners gradient amplifiers and insist that manufacturers stay below specified limits.

- Radio waves: every MRI scanner has a powerful radio transmitter that generates the electromagnetic field which excites the spins. If the body absorbs energy, heating occurs. For this reason, the rate at which energy is absorbed by the body is being limited by the MR sequence parameters.

- Acoustic noise: MRI examinations are characterized by loud noises caused by vibrations generated by rapid switching on and off of the field gradients. Appropriate ear protection is therefore mandatorial for anyone inside the MRI scanner room during the examination.

- Claustrophobia: despite being painless, MRI scans can be unpleasant for those who are claustrophobic. Some modern scanners have larger bores (up to 70 $\mathrm{cm}$ ) and scan times are shorter. This means that claustrophobia could be less of an issue, and additional patients may now find MRI to be a tolerable procedure. Otherwise other strategies may be developed (advanced preparation, visiting the scanner room 
prior to the procedure or, as last resort, sedation).

- Contrast agent: as stated previously, MR contrast agent precautions are limited to patients suffering kidney dysfunctions and/or history of anaphilactoid reactions.

Nowadays one of the main fields of clinical application of MRI is the diagnosis of tumors, i.e. in Oncology. MRI provides detailed information about brain tumor anatomy, cellular structure and vascular supply.

The conventional definition of "brain tumour" refers to an abnormal and uncontrolled cell division originating from brain parenchyma, the meninges, the pituitary gland or of the osseous intracranial structures that can directly or indirectly affect brain tissue. In the United States between 2005 and 2009 it is estimated that there have been 13,000 deaths/year as a result of brain tumours [1]. Based on the location, most tumors will present with clinical signs and symptoms related to mostly mass effect such as headache, seizures, and neurological and cognitive deficits.

Treatment of a brain tumour includes surgery, radiation therapy, chemotherapy, immunotherapy, or a combination of the three. Surgery is the treatment of choice for accessible brain tumors [2]. Accessible tumours are those which can be surgically removed without causing severe neurological impairment. The goal of surgery is gross total resection with functional preservation, providing a chance for other treatments, such as newer forms of radiation therapy.

MRI represents the most widely used tool for a noninvasive and radiation free evaluation of human tumors $[3,4]$. Improvements in anatomical resolution (i.e. use of high-field MRI) and new imaging techniques (i.e. MR spectroscopy, Perfusion-Weighted Imaging, DiffusionWeighted Imaging and functional Magnetic Resonance Imaging) have opened new frontiers in radiology, allowing evaluation of molecular, physiological and metabolic characteristics of a lesion and both before and after treatment [4].

MRI is used for 1) detection and characterization of the lesions (diagnosis); 2) assessment of local involvement and/or distant extension of the disease (staging); 3 ) evaluation of morphological/biological changes over time and response to treatment (follow-up). It also offers invaluable information concerning brain anatomy, tumoral growth pattern and in the selection of patients for intraoperative cortical stimulation, in functional neuro-navigation and in the feasibility of surgical treatment.

\section{MRI TECHNIQUES}

\subsection{Functional Magnetic Resonance (fMRI)}

Functional Magnetic Resonance Imaging (fMRI) is a relatively new technique that builds on the basic proper- ties of MRI to measure quick and tiny vascular (capillary) changes that take place during brain activity. In 1990 Ogawa and Lee at the AT and T Bell Laboratories working on rodents discovered that the oxygenation level of blood acts as a contrast agent in MR images and demonstrated that signals received from vessels were altered by drug-induced changes in blood flow from the brain. They used this type of dynamic information as source of evaluation of cortical function. Advances in computing power and signal processing have boosted its capabilities, providing a wealth of information for neurologists, psychologists, and surgeons. fMRI studies are now capable of providing not only an anatomical view of the brain, but a functional recording of actual brain activity. Since its clinical introduction in 1992, it has become the most widely used imaging technique for studying brain function in humans and its abilities have opened the door to direct observation of cognitive activities. Yet, the technique is under ongoing constant refinement. The big advantage of fMRI is that it doesn't use radiation like CT and Positron Emission Tomography (PET). Technically speaking, fMRI has virtually no risks. It can evaluate brain function safely, noninvasively and effectively. fMRI is easy to use, and the images it produces are very high resolution (as detailed as 1 millimeter). Therefore the key of its success lies in its high spatial/temporal resolution and the lack of biological invasiveness. The biggest drawback is that fMRI, exploiting oxygen changes at a micro-capillary level as source of contrast, represents an indirect source of brain mapping function. It doesn't measure and doesn't rely strictly on synaptic activity, which is critical to mental function. Each area of the brain studied in fMRI is made up of thousands of individual neurons, each of which might have a unique story to tell. Because certain areas of the brain that "light up" on fMRI may represent a number of different functions, it's hard to tell exactly what kind of brain activity is being represented on the scan.

fMRI is most commonly performed using the so called "blood oxygenation level dependent" (BOLD) contrast to study local changes in deoxyhemoglobin concentration in the brain. BOLD imaging takes advantage of inherent differences between oxygenated and deoxygenated hemoglobin. Each of these states has different magnetic properties, diamagnetic and paramagnetic respectively, and produces different local magnetic fields. Due to its paramagnetic properties, deoxy-hemoglobin has the effect of suppressing the MR signal, while oxy-hemoglobin does not. The cerebral blood flow refreshes areas of the brain that are active during the execution of a certain task with oxygenated blood, thereby changing the local magnetic susceptibility and the measured MR signal in active brain regions. A series of properly acquired MR images can therefore be used to study changes in blood 
oxygenation which, in turn, can be used to infer brain activity. Oxy and Deoxyhemoglobin are the link between neuronal activity and fMRI maps. The underlying evoked hemodynamic response to a neural event is typically referred to as the hemodynamic response function (HRF). The increased metabolic demand due to neuronal activity leads to an increase in the inflow of oxygenated blood to active regions of the brain. Since more oxygen is supplied than actually consumed, this leads to a decrease in the concentration of deoxy-hemoglobin which, in turn, leads to an increase in signal. This positive rise in signal has an onset approximately 2 seconds after the onset of neural activity and peaks $5-8$ seconds after that neural activity has peaked.

In patients with cerebral tumors, fMRI is primarily used for neurosurgical planning for preoperative risk assessment, especially in lesions located in close proximity to the eloquent cortex [5,6]. Sunaert S. (2006) identified three main goals of presurgical functional MRI: 1) determine the risk for eventual neurological deficits, by identifying the distance between the margin of planned tumour resection and the eloquent/essential functional areas [7]. It seems that the risk for postoperative loss of function, as tested using functional MRI, is significantly lower when the distance between tumour periphery and BOLD activity is $10 \mathrm{~mm}$ or more [8]; 2) select patients for intraoperative cortical stimulation [6]; 3) provide guidance for functional neuronavigation, based on preoperatively acquired structural information [9].

Functional data can be registered to high-resolution MR morphological images acquired in the same session, enhancing the possibility to identify functional foci within specific anatomic structures. The precise co-registration and data transfer of functional MR imaging into the surgical field allows the preservation of brain functions.

\subsubsection{Studies Design}

There are several common objectives in the analysis of fMRI data. These include localizing regions of the brain activated by a task, determining distributed networks that correspond to brain function and making predictions about psychological or disease states [10]. In neurosurgery fMRI is used to assess the risk of post-operative functional deficits and, during the surgical procedure itself, to identify brain regions that are involved in various functions (i.e. sensory-motor, tactile, language, vision and auditive) [11]. Preoperatively, functional images provide a three-dimensional cortical roadmap that may help the neurosurgeon in the procedure; intraoperatively it may guide the neurosurgeon within the limited view of the craniotomy. fMRI targets defined areas activated by specific stimuli. The choice of the fMRI task is therefore very important. Block design and event-related design are the two main experimental designs used in a fMRI exam. Block design is the most commonly used and compares signal differences between two states, usually a rest and an active condition. Event-related fMRI requires a more complex approach, with robust experiment design and analysis that avoid the so called "habituation effect"; it also allows evaluation of higher congnitive functions. There are various parameters that have to be considered when modeling an experimental design: the simplicity and reprodicibility of the task, the possibility to monitor the experiment during the exam and, most important, the selection of the proper paradigm that may activate a specific brain area involved by or close to a cerebral lesion [10]. The drawback is that up to date no general agreement has been reached within the scientific community in terms of standardization of task designs, i.e. which may be the best task or how many tasks should be used during the MR procedure, especially when evaluating higher cognitive functions. Thus, the reproducibility and sensitivity of fMRI tests may be increased by using a multifunction task battery. The use of multiple paradigms during the scanning session can enhance the specificity of cerebral mapping, optimizing presurgical planning. Another important feature that needs to be carefully considered is the type of analysis of functional (raw) data. A standard fMRI study gives rise to massive amounts of noisy data with a complicated spatio-temporal correlation structure. Statistics plays a crucial role in understanding the nature of the data and obtaining relevant results that can be used and interpreted by neuroscientists. A pragmatic approach must be observed, including strict quality control and method, which are needed to determine if the observed signal changes correlate with the BOLD response curve elicited by the task. This may in turn avoid the chance of false-positive activations. A threshold for statistical significance is required and it can be very variable, therefore the choice of the most adequate is crucial. Last but not least, the patient's compliance is a critical feature that must be assessed and tested before the procedure. Patient cooperation is essential and intensive pre fMRI testing must be performed in order to achieve a good quality map. Movements' artefacts or a wrong comprehension of tasks may alter or change functional results.

\subsubsection{Clinical Applications}

The most common applications of presurgical fMRI are sensori-motor and language mapping [6,11]. The diagnostic objectives are primary motor (M1) and sensory (S1) cortex location, imaging of somatotopy and detection of neuroplastic changes. M1 and S1 targetting can now be performed almost on routine basis. The fMRI signal of motor paradigms is robust and the tasks are easily feasible and repeated. In particular, motor tasks 
are used to localize the hand, foot and mouth cortical representations for cerebral lesions in or near the Rolandic areas. They include:

1) Finger tapping, self-paced clenching of the fist or sponge squeezing (hand localization);

2) Foot dorsal flexion and extension (foot localization);

3) Lip contraction and extension (mouth localization).

Excellent anatomical representation and central sulcus landmark is thus easily accomplished, even in cases of infiltrative lesions that distort normal anatomy; good correlation between the clusters of activation and the areas of representation of the movement.

Tactile tasks as palm brushing, compressed air puffs to the hand and scratching the ventral surface of the hand may be added in order to localize S1.

Visual tasks for lesions in or near the occipital lobes need intermittent photic stimulation and various projected patterned stimuli that are presented by MR compatible hardware devices and systems that must be part of the tools available in the MR suite.

Mapping of cortical eloquent areas is much more complex as the function itself implies a higher cortical involvement. The diagnostic objectives include mapping of the speech centers (Broca, Wernicke), determination of the speech dominant hemisphere and neuroplastic changes detection. Presurgical fMRI planning in patients with lesions near or involving eloquent cortex and the corresponding functional networks has improved in the past few years as scientific knowledge in the field has boosted both because of the higher number of high field scanners available worldwide and because of a growing interest of neuroscientists for the technique [12-14]; every day an increasing number of neurosurgeons rely on this procedure in order to determine hemispheric language dominance and localize cortical language regions [13]. Protocols include multi task battery tests; as stated, no general consensus has been reached on the number and type of paradigms to be used. Usually the procedure includes verb and word generation, word reading, object naming, semantic tasks (for instance, production of nouns or verbs starting with a given letter) or auditory noun presentation with a required category response. This leads to a good mapping of the anterior-posterior networks and excellent representation of the pattern of lateralization (Figure 1).

Patient cooperation is essential and pre-fMRI training is very important. The application of fMRI tasks to map cognitive functions is always complex, because it implies simultaneous/sequential activation and inhibition of multiple regions of the brain, with the real possibility of false-positive activity. Moreover, activation in fMRI during a specific task can be distributed not only in the expected brain regions but in other secondary areas that may also involved in that task. Another important feature

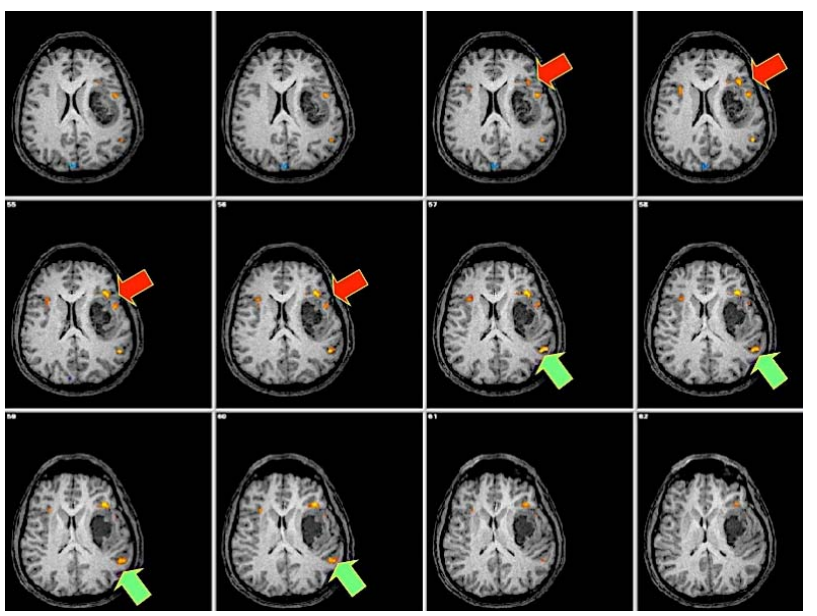

Figure 1. fMRI cerebral mapping in high grade glioma involving the left frontal and temporal lobe. Excellent representation of the anterior (Broca, red arrow) and posterior (Wernicke, green arrow) verbal network during a multi-paradigm task test. The clusters of activation lie close to the antero-lateral margin of the tumor.

to remember is brain plasticity, that leads to a topographic reorganization of brain functions in patients with cerebral lesions [15]. The use of multifunction task battery maximizes the reliability of the targeting and allows a safe lesional margin resection an improved outcome and lower morbidity. fMRI has therefore gained increasing popularity and is rapidly taking the place of the invasive Wada test when hemispheric dominance is needed. The literature has shown a strong correlation between fMRI and the Wada test for determination of hemispheric language dominance [16,17].

\subsubsection{Conclusions}

Although fMRI technique can not completely substitute other forms of tests (i.e. intraoperative cortical stimulation and the Wada test), in the near future it certainly provide valuable adjunctive information when a specific functional evaluation is required.

Sensory, motor and verbal task are reliable. They are easily carried out with standardized protocols; yet, neurosurgeons must bear in mind that especially when dealing with verbal or other higher cognitive functions mapping-complex functions may need too many paradigms, leading to a time-consuming procedure that may not be easily accomplished by the patient. Moreover, different tasks may have a different specificity and there are issues that remain unsolved when trying to locate a single specific function or area. Neuropsicological test are therefore essential and must be part of the working schedule.

fMRI is therefore useful for presurgical planning in patients with lesions near sensory, motor, and language areas. It is safe, easily performed, it may evaluate multiple functions, and has excellent temporal and spatial 
resolution. Yet it may not be considered the alternative to direct cortical stimulation (DCS). Overall reliability for both types on mapping is high (sensibility $94 \%$, specificity $89 \%$ ), but more work needs to be done. The lower rate in patient sensitivity $(77 \%)$ for the language areas as compared to sensory-motor figures presumably reflects tumor-related receptive and expressive aphasias as well as related cognitive losses. In the presurgical planning, the functional evaluation of verbal capacities is especially useful for the determination of hemispheric dominance. The calculation of lateralization index (LI) allows to define the precise localization of the functional areas related to the tumor. Other advantages may come along with integration with other imaging modalities as Diffusion Tensor Imaging (DTI) and Magnetic Resonance Spectroscopy (MRS).

Stronger efforts are now made to expand fMRI capabilities, in order to shift from mere structural (anatomical) connectivity to effective functional connectivity that may directly target focal brain areas.

\subsection{Diffusion Weighted Imaging (DWI) and Diffusion Tensor Imaging (DTI) Principles}

Diffusion-Weighted Imaging (DWI) is a novel technique that reveals the microscopic structure of a tumour and yields information about the trend of malignancy of the lesions in terms of cellularity analysis [18]. The first diffusion MRI images of the normal and diseased brain were made public in 1985. At that time diffusion MRI was a very slow method, very sensitive to motion artifacts. It was not until the availability of ultraist EchoPlanar Imaging (EPI) technique on clinical MRI scanners that diffusion could really take off in the early 1990s. This move into the clinical field was the result of an intense and fruitful collaboration between Denis Le Bihan and Robert Turner, both researchers at NIH. With Turner's unique expertise in gradient hardware and EPI gained during the years he spent with Peter Mansfield, they were able to obtain the first EPI images also with the help of colleagues from General Electric Medical Systems (Joe Maier, Bob Vavrek, and James MacFall). With EPI and diffusion, images could be obtained in a matter of seconds and motion artifacts became history (of course, new types of artifacts came along later). Since then, DWI has been extraordinarily successful. In the mid 1990s its main clinical application has been in the study and treatment of neurological disorders, especially for the management of patients with acute stroke. As a matter of fact, DWI is capable to show within a couple of minutes the exact localization and extent of an acute infarct, even before a standard morphological MRI does. DWI is now a well established method for diagnosing cerebral ischemia and it is a routine protocol in most institutions that perform neuroimaging. Moreover it is currently gaining popularity in the investigation of other brain disorders involving cerebral white matter such as epilepsy, multiple sclerosis, brain abscesses hypertensive encephalopathy and, lately, brain tumours. This approach includes qualitative and quantitative analysis $[19,20]$.

Diffusion, or Brownian movement, denotes the random motion of molecules. All molecules exhibit such motion at temperatures greater than absolute zero. Diffusion is termed isotropic if the motion is equal in all directions. Yet, the motion encounters different obstacles in the living environment (cell membranes, proteins, macromolecules, fibers), which vary according to the tissues and certain pathological modifications (intracellular edema, abscess, tumors...).

Stated simply, the displacement of water molecules can be summarized into three different types of freedom of movement:

1) Free diffusion: the water molecules displace freely in all spatial directions. A typical example of this corresponds to fluids such as cerebrospinal fluid;

2) Restricted isotropic diffusion: water molecule displacement is restricted, in whatever spatial direction, by numerous obstacles (proteins, cells). Example: abscess, tumor at high cell density;

3) Restricted anisoptropic diffusion: certain structured tissues create obstacles that orientate the motion of the water molecules (tendency to displace themselves in one or several particular directions). Diffusion is only restricted in certain spatial directions. Example: nerve fibers (organization in bundles of axons running in parallel, with concentric layers of myelin restricting transversal diffusion).

Essentially, extracellular water is the main object of exploration in diffusion imaging. Diffusion data thus provides indirect information about the structure surrounding these water molecules.

Imaging and interpretation of water diffusion have improved with the development of diffusion tensor imaging (DTI). Based on DWI principles, it took off in the early 90s along with EPI technical developments. DTI allows direct in vivo and non invasive examination of aspects of the tissue microstructure. It takes advantage of diffusion anisotropy to provide excellent details of the brain, enabling mapping of the orientation of the whitematter tracts and providing models of brain connectivity. DTI enables the measurement of the restricted diffusion of water in tissue in order to produce 2D or 3D neural tract images instead of using this mathematical data solely for the purpose of assigning contrast or colors to pixels in a cross sectional image.

Image interpretation and analysis is crucial. Diffusion tensor measurements result in a rich data set. Diffusion anisotropy may be measured by applying simple or com- 
plicated mathematical formulas. However, an easy and common way to summarize diffusion measurements on diffusion tension images is to calculate parameters for overall diffusivity and for the degree of anisotropy.

These parametrs include the apparent diffusion coefficient (ADC), which is a measure of the magnitude of molecular motion; fractional anisotropy (FA), which is the measure of the directionality of the molecular motion of water; relative anisotropy (RA), or the ratio between anisotropic and isotropic portions of the diffusion tensor; and the volume ratio (VR), which expresses the relationship between the diffusion ellipsoid volume and that of a sphere, the radius of which is the averaged diffusivity [19].

Maps of both FA and RA may be presented as grayscale images. Regions of interest (ROI) are placed on both maps to calculate diffusivity and FA.

FA is sensitive to low values of diffusion anisotropy; VR is sensitive to high values of diffusion anisotropy; and RA is linearly scaled for different levels of anisotropy. Both FA and RA vary from 0 (isotropic) to 1 (anisotropic). The values of measurements in pediatric brains markedly differ from those in adult brains; values vary with increasing age. Mean diffusivity is approximately $0.7 \times 10^{-3}$ in adults and $2 \times 10^{-3}$ in neonates. Because anisotropy is greater in ordered structures, such as myelinated axons, DTIs provide useful information regarding the myelination of white matter.

In many pathologic conditions, FA and ADC vary because of altered diffusivity and disorganization of the white-matter fibers, leading to decreased anisotropy. These measurements may become abnormal even before the lesion is morphologically apparent on conventional MRIs and may therefore help in early detection and in defining the extent of these lesions.

FA and ADC may vary independently. This may be explained by the fact that damaged or malformed brain has glia and neurons, respectively that have different effects in terms of cellularity and diffusion. They have enough cell density to prevent effects on ADC; however, because of the disorganization, they may cause diverse FA reduction.

\subsubsection{Clinical Applications \\ 1) DWI}

Tissues with high cellularity have a low ADC because the mobility of water protons is impaired. In tumours, ADC values are usually highest in cystic or necrotic areas. Solid portions have different patterns, related to the degree of malignancy [18]. Low-grade gliomas tend to have a higher ADC compared with high-grade lesions. The ADC value seems to be inversely related to tumour cellularity and grading. In morphological MRI, after contrast media administration, the tumoral enhancing areas are usually considered the foci of higher malignancy and the target of surgical excision. However, it is now commonly recognized that T2-FLAIR hyperintense and/or nonenhancing tissue may imply a high grade malignancy as well; this has opened new fronteers in neuroimaging, setting the pace for DWI application in neurooncology. In a recent study has studied the ADC values in patients with malignant supratentorial astrocytoma as prognostic tool [21].

ADC may be used to target the sites of highest cellularity within heterogeneous tumours; identifying these areas may be important for diagnosis and prognosis and can be useful to characterize highly cellular versus low cellular components. DWI therefore represents a promising tool for preoperative grading, postoperative assessment of glial tumours, differential diagnosis between recurrence or radionecrosis or in the assessment of therapeutical response injury. DWI now forms part of today's clinical MR protocols in areas such as head, neck, body, muscolo-skeletal and breast, adding information about tissue composition to conventional morphological sequences, with the advantage of need neither contrast media injection nor ionizing radiation. Conversely, DWI has not still exploded its full potential; it is currently being used as detector of pathology, but new oncological applications as prediction of tumor response or early post treatment monitorization have to be completely explored.

\section{2) DTI}

The goal of maximal safe resection is hard to achieve without detailed information on the underlying functional areas, white matter bundles and their relationship with the pathologic components. The advent of DTI has revolutionized the study of brain connectivity in the living human brain.

Tractography potentially solves a problem for a neurosurgeon in terms of minimizing functional damage and determining the extent of diffuse infiltration of pathologic tissue to minimize residual tumor volume. In this way, tractography facilitates preoperative planning. Tractographic images may help to clarify whether a tumor is compressing, abutting, or infiltrating the contiguous white-matter tracts. However, no consensus has been reached about an appropriate criterion standard for assessing the accuracy of DTI, and this technique is primarily investigational at present

Tractography has allowed visualization of white matter tracts and is becoming an essential tool for neurosurgical planning [22-25] and postoperative follow-up of surgically treated brain tumours [26] and vascular malformations. White-matter tract visualization by means of diffusion anisotropy maps has had a major impact on neurosurgical case management. Knowledge of the topography, integrity, and degree of involvement of the white matter fibers plays an important role in pre-opera- 
tive evaluation.

DTI provides information about the integrity, displacement and/or interruption of white matter tracts in and around a tumour; it may also detect widening of fiber bundles due to edema or tumour infiltration [24,27, 28]. White matter tracts are characterized as follows: displaced, if they maintain normal anisotropy relative to corresponding contralateral tract but are locate in an abnormal location or abnormal orientation according to the standard color code, edematous, if they maintain normal anisotropy and orientation but show obvious increased signal intensity on T2 images, infiltrated if they show reduced anisotropy yet remain visible on the orientation maps, and degenerated if the anisotropy is significantly reduced as such there are no identifiable tracts on the orientation maps. For tracts characterized as infiltrated is difficult determine whether anisotropy was reduced as a result of vasogenic edema, tumor infiltration or a combination of these factors [29]. Such a distinction is to date extremely difficult if not impossible only by DTI. DTI results may vary enormously depending on the chosen method of analysis. The most common approach is streamline tractography which is closely related to an earlier method for visualization of tensor fields known as hyperstreamlines. This method produces as output discrete curves or trajectories that are also called "tracts", "fibers", "traces", etc. Several computational methods can be used to perform basic streamline tractography. These include Euler's method, second order Runge-Kutta (also known as the midpoint method), and fourth order Runge-Kutta. Another popular method is called FACT. Other techniques include probabilistic tractography that outputs connection strengths or probabilities, optimization methods that use graph theory or physical models region-growing and wavefront evolution methods, tractography using advanced models for fiber crossings, and tractography "meta-analysis" methods that perform clustering or fit more sophisticated tract models [30]. All these methods can produce false positive and false negative results; however it is important to note that clinical validations of streamline tractography have demonstrated accurate reconstructions (true positive results). Tract endpoints, especially of the corticospinal or motor tract, have been compared to electrocortical stimulation during neurosurgery with good correspondence [31]. In a study of 238 neurosurgical patients with gliomas involving the motor tract, where patients were randomly assigned to study or control groups, the use of DTI was shown to increase survival and reduce postoperative motor deficits [32].

\subsubsection{Clinical Applications}

Correct pre-operative identification of white matter tracts is at least as important as the identification of eloquent cortices in fMRI. It is impossible to identify and separate by visual inspection white matter tracts, such as the cortico-spinal tract (CST-the main motor tract) or the thalomo-cortical tract (the main sensory tract) as they traverse the corona radiata. Diffusion tensor imaging has been advocated as a the most important tool that may precisely localize white matter bundles, minimizing injury to critical functions as motion, language, and vision (Figure 2).

In presurgical sensory-motor planning, the corticospinal and the thalamo-cortical tracts have reached the highest level of standardization. On the other hand, in language mapping the superior longitudinal fasciculus (SLF), the arcuate fasciculus (AF) and the inferior frontooccipital fasciculus (IFOF) are the white matter bundles that have received major validation with DSC.

The heterogeneity of brain tumours in the presence of complex environments (e.g., edema, mass effects) and the inherent heterogeneity of diffusion anisotropy in normal white matter reduce the overall specificity of DTI measures. By using mean diffusivity (MD) and anisotropy measures, several researchers $[30,33]$ have tried to differentiate tumour infiltrated edema associated with high-grade gliomas from purely vasogenic edema associated with metastases and meningiomas. In general, it is assumed that increased cellular densities will decrease the mean diffusivity (MD) and that the MD will be significantly elevated in areas of tissue necrosis. There is a correlations between FA and cell density and proliferation in both astrocytomas and glioblastomas, with higher FA values corresponding to higher cell densities.

Researchers have also tried to use DTI to differentiate between gliomas and edema, which is challenging with

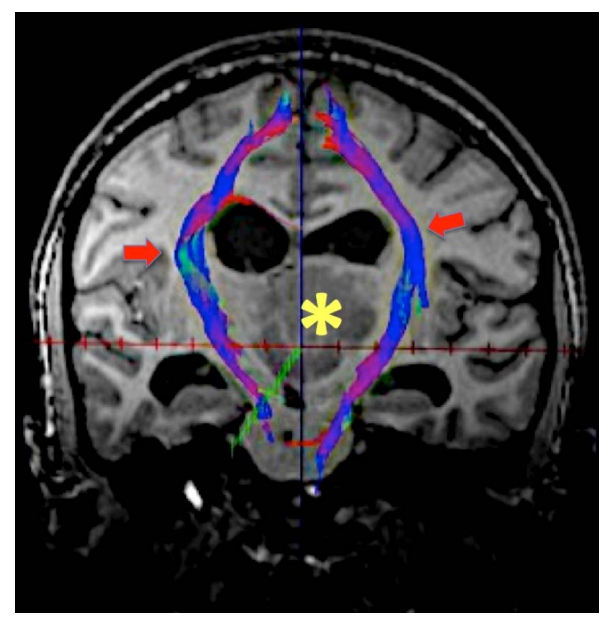

Figure 2. DTI-tractography in patient with anaplastic astrocytoma (yellow asterisk) of the thalami (coronal view). Integrity of corticospinal tracts (red arrows), which are slightly divaricated by tumoral mass effect. No signs of infiltrative involvement. 
conventional MRI techniques methods [30]. Tract orientations appear to be highly affected by infiltrating gliomas, whereas the directions appear to be preserved in regions of edema. Lu et al. (2004) developed a criterion (the Tumor Infiltration Index) based on the relationships between FA and MD to classify neoplasia and peritumoral edema [33]. Another study [29] using DTI has found no significant differentiation between tumor types and edema. The type of edema may also influence the diffusion properties. Morita et al. (2005) showed as a peritumoral edema from high-grade tumors (relative to low-grade) appeared to have higher diffusivity, possibly from the destruction of the extracellular tissue matrix [30].

In conclusion, DTI provides non-invasively crucial information that helps surgeons take proper preoperative decisions: 1) whether to resect or not a lesion, based on evidence of tumoral infiltration of a functional area/ bundle, 2) how much tumoural tissue can be safely removed, 3) the safest surgical entry.

\subsubsection{Issues in DTI Data Interpretation}

Many factors (e.g. cell death, change in myelination, increase in extracellular or intracellular water, etc.) may cause changes in FA. Overall, this difficulty in interpretation of DTI is due to the fact that the scale at which diffusion is measured with DTI is very different from the size scale of individual axons. The tensor model is only able to represent one major fiber direction in a voxel, thus DTI tractography can be confounded by regions of high density crossing fibers. Other factors may confound tractography: partial volume effects, where two types of tissue are present in a voxel, can produce a tensor that represents neither tissue well. Also, crossing, "kissing", and "fanning" fiber tracts are not well represented at the voxel level by the diffusion tensor. Finally, in standard streamline tractography all decisions are made locally, thus errors can accumulate.

Common false positive connections include trajectories from the corona radiata that cross the corpus callosum and trajectories from the corona radiata that cross at the pons and ascend in the corona radiata of the other hemisphere. Common false negatives with DTI tractography are the lateral lip/hand connections of the corticospinal tract and lateral connections of the corpus callosum.

The particular errors depend strongly on the tractography algorithm employed, and on the type of diffusion data used (DTI versus higher-order models). However, to date there is no perfect method, and it is unlikely that perfect tractography is possible.

\subsubsection{Conclusions}

Diffusion in structured tissue, such as white matter, is anisotropic. DTI can be used to measure anisotropy per voxel and provides the directional information relevant for magnetic resonance tractography or fiber tracking in vivo. The recent development of DTI allows for direct examination of the brain microstructure; DTI has therefore become a useful tool for investigation of brain disorders such as stroke, epilepsy, MS, brain tumors, and demyelinating and dysmyelinating disorders.

Although the technique is a powerful tool to study and visualize white matter, its demerits lie on the methodological and mathematical foundations, as it must be constantly validated, challenged and developed in terms of acquisition scheme, image processing, analysis, and interpretation, because of inherent artefacts and limitations. Newer algorithms of DTI and Tractography are being developed in order to strengthen the roots and validate the potential of clinical application. In particular, although DTI has no clear "killer application" yet, it has tremendous potential. In the coming years, development in this area will more rapidly create these $3 \mathrm{D}$ white matter "roadmaps" of the brain, and be able to, for example, map white matter structural deviations of a specific individual relative to a standard template built from various patient populations.

At any rate, when combined with functional brain mapping, DTI now provides an efficient tool for comprehensive, no invasive, functional anatomy mapping of the human brain, and efforts have been made in recent years to integrate DTI data with neurosurgical navigation systems [25,28,31,32]. DWI and DTI, in addition to conventional MRI, can help to characterize tissue by distinguishing solid enhancing tumour, no contrast enhancing lesion, peritumoral oedema, and necrotic or cystic regions from normal surrounding brain tissue [18]. Tractography combined with functional MRI may potentially help in preoperative planning of brain tumours by mapping areas of active infiltration opening the way to non invasive investigation of the connections between axonal fibers in vivo and functions of different brain regions.

\subsection{Perfusion Weighted Imaging (PWI)}

Perfusion Weighted Imaging (PWI) is a relatively new MR technique that provides information on the hemodynamic status of the tissue and can detect impaired perfusion in both the ischemic core and the surrounding brain regions, thereby complementing the information derived from DWI. PWI displays microvascular haemodynamics. Blood flow in the cerebral vascular network can be measured by quantitation of haemodynamic parameters like the regional cerebral blood flow $(\mathrm{rCBF})$ and the regional cerebral blood volume (rCBV). Based on these premises and knowing that microvascular proliferation is 
one of the most important histopathological criteria when determining the malignancy of gliomas, PWI has rapidly been established a valuable tool for assessing tumour angiogenesis.

PWI permits no invasive in vivo study of tumour vascular hyperplasia and capillary permeability, which are important biological markers of malignancy, grade and prognosis, particularly in gliomas. Two perfusion techniques are used: arterial spin labelling (ASL, without contrast media) and dynamic susceptibility contrast (DSC) enhanced perfusion imaging. The ASL signal uses blood normally circulating in the vascular bed as endogenous tracer. Blood is labelled in special sequences for the identification and quantification. DSC technique is know as contrast agent bolus tracking and employs exogenous tracer (i.e. Gadolinium) as paramagnetic contrast media administered by rapid infusion during the acquisition of ultrafast MR sequences. Gadolinium contrast agent passes into the arterial and capillary circulation and produces signal changes in vessels and surrounding tissues. This imaging modality is based on T2 susceptibility effects of Gadolinium, which alters the magnetic susceptibility of blood, creating a sharp drop in signal intensity in T2 weighted images; this in turn is proportional to the amount of Gadolinium present at the time and to the cerebral perfusion. Under a pathological perfusion (i.e. hyperacute ischemic lesion) the signal decrease is attenuated or delayed, or varies in relation to grading (as in brain tumours). Important quantitative parameters that characterize PWI include: Mean Transit Time (MTT, the average time spent by the blood in the cerebral vascular bed) and Time To Peak (TTP, the time taken from the start of bolus injection to the maximum signal intensity) Yet, the most robust quantitative parameter is relative cerebral blood volume (rCBV), which represents tumour angiogenesis.

\subsubsection{Clinical Applications}

Accurate cerebral blood flow (CBF) measurements in clinical patients who have neoplastic (and vascular) cerebral disease remain a serious imaging challenge, regardless of magnetic field strength or even imaging modality. Quantitative CBF values are frequently considered to weigh the risks and benefits of surgical versus medical (chemotherapy) management. Aside from MR, this frequently necessitates more invasive and costly imaging tests, such as single-photon emission computed tomography (SPECT) or PET. PWI allows better estimates of biological activity and aggressiveness in brain tumours, more rapid visualization and a better prediction of the course of the disease than conventional MRI diagnostics. Several studies [34-36] have demonstrated a strong positive correlation between tumour rCBV and astrocytoma grading. Relative cerebral blood volume (rCBV) ratios were calculated on the solid portion of the tumour, on peritumoural area, as well as on the contralateral normal white matter, respectively. The results showed that higher rCBV ratios were present in both solid portions and peritumoural regions of anaplastic gliomas, but not in low grade gliomas. PWI should be integrated in the diagnostic work-up of non-enhancing gliomas in order to predict grading. Good correlations between perfusion parameters and tumor grade have also been established using dynamic T1-weighted MR permeability imaging. In a group of 22 untreated gliomas, Roberts et al. showed a strong correlation between tumor grade and permeability $(\mathrm{r}=$ 0.76) [37]. A similar observation was made in a study by Provenzale et al. about first-pass tumor permeability using dynamic susceptibility MR perfusion in a series of 22 patients, which included both treated and untreated cases [38]. In this study, there was overlap in first-pass permeability (K2) values between different tumor grades. The data suggest that a cut off of 0.03 in permeability separates low-from high-grade tumors, with a positive predictive value of $90 \%$ and negative predictive value of $75 \%$.

\subsubsection{Conclusion}

MR perfusion main advantages are relative non invasiveness as well as quali and quantitative capability of assessment of ischemic or tumoral hemodynamics. PWI in the latter aids in grading and better differentiation in diagnostics as well as in pre-therapeutic planning. Thanks to this novel technique the course of treatment, both after chemo-as well as radiotherapy in combination with surgical treatment, may be optimized. Preoperative grading of brain tumors can be used to guide the surgeon to perform biopsy on the most aggressive portions of a tumor. PWI allows better estimates of biological activity and aggressiveness in low grade brain tumors, and in the case of WHO grade II astrocytoma showing anaplastical transformed tumor areas, allows more rapid visualization and a better prediction of the course of the disease than conventional MRI diagnostics. It is also possible that PWI could be used to more accurately delineate tumor margins, in order to better plan resection and radiation treatment of brain neoplasms. MR perfusion imaging may also be valuable in distinguishing radiation necrosis from tumor recurrence, thus sparing patients from unnecessary treatment. Finally, MR perfusion methods, as a surrogate marker for treatment outcome, are likely to play a central role in the development of new antiangiogenic compounds. As advances in MR technology take place, the role of MR perfusion in the care of neuro-oncologic patients is likely to increase, and may eventually permit reliable, noninvasive assessment of a patient's prognosis and response to therapy [39]. More efforts are to be made in order to improve ASL techniques that al- 
low measuring perfusion without contrast media administration; the advantages are that completely noninvasive absolute cerebral blood flow (CBF) measurements are possible and that multiple repeated measurements can be obtained to evaluate one or more interventions. Recent technical advances have improved the sensitivity as well as its spatial resolution of ASL perfusion MRI, and increasing use is expected in the coming years.

\subsection{Proton Magnetic Resonance Spectroscopy ( ${ }^{1}$ H-MRS)}

In vivo proton Magnetic Resonance Spectroscopy $\left({ }^{1} \mathrm{H}-\right.$ MRS) is a non-invasive technique that allows detection of metabolic cerebral tissue properties of living systems. MRS has been used for decades for in vitro assessment of tissue and cellular biochemistry, prior to the introduction of in vivo studies in the 1980s. MRS has been applied in a large number of clinical studies over the last decade and it is now fully integrated into the clinical MRI diagnostic procedures.

High-resolution spectroscopic imaging can contribute to pre-therapeutic grading and characterization of intraaxial lesions, especially when the routinely performed MR sequences do not provide accurate differential diagnoses. Sibtain et al. (2007) defined three situations in which MRS increases diagnostic confidence [40]: distinction of high-grade gliomas and metastases from abscesses; assessment of tumour recurrence as opposed to radiation necrosis; identification of radiologically atypical meningioma as a true meningioma. MRS also provides useful information for tissue characterization in the diagnosis and treatment of brain tumours, necessary for selecting the most appropriate treatment, directing the therapy, targeting the biopsy to the most active area of the tumour or help the neurosurgeon in the resection of lesion.

\subsection{1. ${ }^{1} \mathrm{H}$-MRS Sequences}

Clinical ${ }^{1} \mathrm{H}-\mathrm{MRS}$ methods include single-voxel spectroscopy (SVS), multi-voxel 2D and 3D chemical shift imaging (2D and 3D CSI). Single-voxel spectroscopy produces a single spectrum from a single voxel, usually in the order of $2-8 \mathrm{cc}$ in volume. Multi-voxel chemical shift imaging (CSI) covers a broader area with smaller voxels, typically $1 \mathrm{cc}$ in volume each. CSI can be displayed as spectral maps, coloured metabolic images, and individual spectra (Figure 3).

The choice of SVS or CSI should be tailored to each particular case. SVS is not optimal for lesion that are too large to be completely contained within the voxel or if the lesion is too small to affect the spectrum from the 2 cc voxel. $2 \mathrm{D}$ and $3 \mathrm{D}$ CSI allow better coverage and higher spatial resolution at the expense of longer acquisition times.

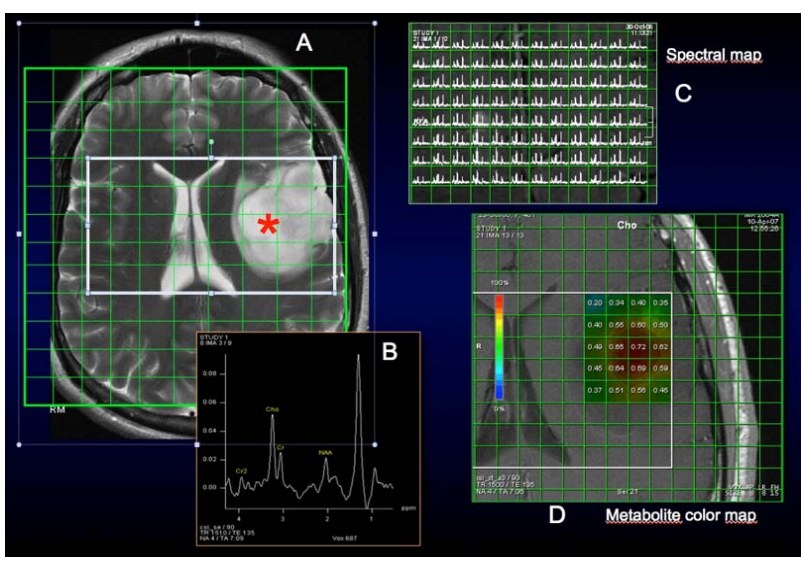

Figure 3. Magnetic Resonance Spectroscopy (MRS). (A) 2D CSI MRS voi (volume of interest, white frame) in patient with oligodendro-glioma (red asterisk) involving the inferior frontal and external capsulae region; (B) MR spectra of a single voxel within the voi shows elevation of Choline, NAA reduction and high peak of lipids, indicative of malignancy; (C) Complete spectral map, showing the whole metabolic pattern within the lesion; (D) Metabolic color map showing Choline (Cho) distribution within the tumor.

\subsubsection{Clinical Applications}

MRS displays brain metabolites in the form of spectra whose shape basically depends on metabolite concentration. Metabolite concentration has been used to measure chemical markers of neoplastic activity.

The principal metabolites that can be analyzed are: $\mathrm{N}$ acetyl-aspartate (NAA), Choline (Cho), Creatine/phosphocreatine $(\mathrm{Cr} / \mathrm{PCr})$, Glutamate/Glutamine $(\mathrm{Glu} / \mathrm{Glx})$, Myo-inositol (mI), Lactate (Lac) and Lipids (Lip), and are potential discriminates of tumour grade. NAA is an amino acid that is present almost exclusively in neurons. NAA decrease corresponds to neuronal death or injury or the replacement of healthy neurons by other (e.g. tumour) cells. The Cho is involved in cell membrane metabolism. Increased Cho metabolism may reflect neoplastic cell membrane synthesis (cellular proliferation, inflammation or demyelination), and studies have found good correlation between Cho and glial tumor grade [41]. $\mathrm{Cr} / \mathrm{PCr}$ is metabolites involved in energy storage. $\mathrm{Cr}$ is a marker of overall cellular density and has often been used as an internal standard for semi-quantitative evaluation of metabolic changes of other brain metabolites. Glu/Glx is involved in excitatory and inhibitory neurotransmission. MyoInositol is a compound present only in glial cells. It is reduced in case of cellular impairment and it increases in cases of glial activation or proliferation. Lactate is produced under conditions of anaerobic glycolysis. It indicates hypoxic conditions as well as hypermetabolic glucose consumption and in some cases macrophagic invasion. It is not routinely detectable in healthy brain. Finally, free lipids usually correlate to the extent of tissue necrosis. The presence of Lac and Lip in brain tu- 
mours reflects anaerobic glycolysis and tissue necrosis, respectively.

To distinguish tumours from other brain lesions were studied chemical ratios between $\mathrm{Cho} / \mathrm{Cr}$, Cho/NAA, $\mathrm{Lac} / \mathrm{Cr}$. Specifically, a Cho/NAA ratio greater than 1 is considered to be indicative of tumoral growth. Elevated levels of Choline compounds have been repeatedly reported in gliomas $[42,43]$ but not in metastases [44]. Decreased concentration values of choline and $\mathrm{N}$-AcetylAspartate allowed to differentiate gliomas from metastases $[44,45]$, infarctions and abscesses. Meningiomas have been characterized by decreased concentrations of myo-inositol, creatine and choline [43] and an elevated concentration of alanine (Al) [46]. Elevated lipid resonances are reported in lymphomas [47]. Levels of myoinositol were higher in low grade astrocytomas than in high-grade gliomas [48].

\subsubsection{Conclusion}

The potential of in vivo NMR spectroscopy to provide extended biochemical information on clinical settings was demonstrated approximately a decade ago. Its main advantage is a non invasive diagnostic capability to evaluate neurochemical pathways and metabolite quantification. Yet, these advantages are strictly linked to the field strength of the scanner (the higher, the better resolution) and to the scanning technique itself (sequence parameters and processing steps), that may invalidate the results. The quantification of metabolites is highly sensitive to spectral quality and data processing. Baseline distortions, poor water suppression, and even minor inaccuracies in avoiding chemical shifts of metabolites may result in substantial systematic errors in data quantification. Is these biases are being considered and taken into account when performing MRS, its benefits and added value if compared to conventional MRI has been demonstrated to significantly increase the proportion of correctly diagnosed cases [49,50] and Stadlbauer et al. (2006) comparing (at a field strength of $1.5 \mathrm{~T}$ ) quantitative MRS imaging with MRS-guided stereotactic biopsies reported good results for preoperative grading of gliomas [51].

\subsection{Conclusion}

Imaging, clinical and neuropsychological data must be integrated in order to assess and rule out the factors that help predict the postoperative outcome. A special emphasis must be given when DTI and tract information (displacement of the fibers relationship with the tumor) is included in the surgical algorithm. Brain tumors can affect functional cortical gray matter and white matter tracts. A good neurological outcome following the resection of infiltrative tumors requires a detailed preoperative understanding of the anatomical relationship with func- tional areas and adjacent cortical white matter tracts. This is of utmost importance when the tumor develops in or near a functional cortical area in the dominant hemisphere, be it motor, sensory, or cognitive. Understanding the location of the lesion in relation to surrounding functional tissue is a vital element in building an efficient surgical plan. The purpose of multiple mapping techniques is to delineate functional areas at best, so they can be preserved during surgery. Maximal tumor resection was shown to be an element closely related to patient survival and improved clinical status of patients on longterm, indicating a main predictor of clinical course. The present data show that tumors that invade and infiltrate functional cortical areas may also include underlying intact white matter tracts within the mass. The rate of unfavorable deficits occurs in $12.5 \%$ of patients and has largely improved when multiple mapping techniques are being used postoperatively. Yet, although they minimize the risk of injury to the functional cortex or to the adjacent tracts, the methods do not guarantee the absence of postoperative dysfunction. These deficits may be caused by subcortical areas invasion, retraction, edema or ischemia. However, based on the results of this study, the risk of harming a previously intact patient during awake craniotomy remains low.

\subsection{Final Remarks}

MR imaging of brain tumours has shown substantial improvements during the past few years, both in terms of high quality morphological imaging (with the use of high-field magnets) and in the shift towards functional and molecular imaging. The integration of functional, metabolic and structural MRI information offers standardized preoperative maps of multiple critical functions to facilitate assessment of surgical risk, planning of surgical routes and direction of conventional, intraoperative electrophysiological procedures. These new imaging applications add greater specificity to a no invasive approach of cerebral tumours and help the neurosurgeon in the assessment of resection and function preservation.

\section{REFERENCES}

[1] Central brain tumor registry of the United States (2012) http://www.cbtrus.org

[2] Sunaert, S. (2006) Presurgical planning for tumor resectioning. Journal of Magnetic Resonance Imaging, 23, 887905. doi:10.1002/jmri.20582

[3] Westbrook, C. (2010) MRI at a glance. Wiley-Blackwell, Hoboken.

[4] Young, G.S. (2007) Advanced MRI of adult brain tumors. Neurologic Clinics, 25, 947-973. doi:10.1016/j.ncl.2007.07.010

[5] Haberg, A., Kvistad, K.A., Unsgard, G. and Haraldseth, 
O. (2004) Preoperative blood oxygen level-dependent functional magnetic resonance imaging in patients with primary brain tumors: Clinical application and outcome. Neurosurgery, 54, 902-914.

doi:10.1227/01.NEU.0000114510.05922.F8

[6] Petrella, J.R., Shah, L.M., Harris, K.M., et al. (2006) Preoperative functional MR imaging localization of language and motor areas: Effect on therapeutic decision making in patients with potentially resectable brain tumors. Radiology, 240, 793-802. doi:10.1148/radiol.2403051153

[7] Sunaert, S. (2006) Presurgical planning for tumor resectioning. Journal of Magnetic Resonance Imaging, 23, 887905. doi:10.1002/jmri.20582

[8] Håberg, A., Kvistad, K.A., Unsgård, G. and Haraldseth, O. (2004) Preoperative blood oxygen level-dependent functional magnetic resonance imaging in patients with primary brain tumors: Clinical application and outcome. Neurosurgery, 54, 902-914. doi:10.1227/01.NEU.0000114510.05922.F8

[9] Rasmussen, Jr., I.A., Lindseth, F., Rygh, O.M., Berntsen, E.M., Selbekk, T., Xu, J., Nagelhus, H.T.A., Harg, E., Haberg, A. and Unsgaard (2007) Functional neuro-navigation combined $\mathrm{G}$. with intra-operative 3D ultra-sound: Initial experiences during surgical resections close to eloquent brain areas and future directions in automatic brain shift compensation of preoperative data. Acta Neurochir (Wien), 149, 365-378. doi:10.1007/s00701-006-1110-0

[10] Lee, C.C., Ward, H.A., Sharbrough, F.W., Meyer, F.B., Marsh, W.R., Raffel, C., So, E.L., Cascino, G.D., Shin, C., Xu, Y., Riederer, S.J. and Clifford, Jr., J.R. (1999) Assessment of functional MR Imaging in neurosurgical planning. Brain. American Journal of Neuroradiology, 20, 1511-1519.

[11] Stippich, C., Rapps, N., Dreyhaupt, J., et al. (2007) Localizing and lateralizing language in patients with brain tumors: Feasibility of routine preoperative functional MR imaging in 81 consecutive patients. Radiology, 243, 828836. doi:10.1148/radiol.2433060068

[12] Seghier, M.L., Lazeyras, F., Pegna, A.J., Zimine, I., Dela, V.J., Mayer, E., Annoni, J.M. and Khateb, A. (2001) fMRI on patients with lesions involving language areas: Implications for neurosurgery. NeuroImage, 13, 836. doi:10.1016/S1053-8119(01)92178-0

[13] Savoy, R.L. (2001) History and future directions of human brain mapping and functional neuroimaging. Acta Psychologica, 107, 9-42. doi:10.1016/S0001-6918(01)00018-X

[14] Atlas, S.W., Howard II, R.S., Maldijian, J, Alsop, D., Detre, J.A., Listerud, J., D’Esposito, M., Judy, K.D., Zager, E. and Stecker, M. (1996) Functional magnetic resonance imaging of regional brain activity in patients with intracerebral gliomas: Findings and implications for clinical management. Neurosurgery, 38, 329-338. doi:10.1097/00006123-199602000-00019

[15] Briganti, C., Sestieri, P.A., Mattei, R., et al. (2012) Reorganization of functional connectivity of the language network in patients with brain gliomas. American Journal of Neuroradiology, 34, 1.
[16] Binder, J.R., Swanson, S.J., Hammeke, T.A., Morris, G.L., Mueller, W.M., Fischer, M., Benbadis, S., Frost, J.A., Rao, S.M. and Haughton, V.M. (1996) Determination of language dominance using functional MRI: A comparison with the Wada test. Neurology, 46, 978-984. doi:10.1212/WNL.46.4.978

[17] Knecht, S., Drager, B., Deppe, M., Lohmann, H., Floel, A., Ringelstein, E.B. and Henningsen, H. (2000) Handedness and hemispheric language dominance in healthy humans. Brain, 123, 2512-2518. doi:10.1093/brain/123.12.2512

[18] Chenevert, T.L., Sundgren, P.C. and Ross, B.D. (2006) Diffusion imaging: Insight to cell status and cytoarchitecture. Neuroimaging Clinics of North America, 16, 619632. doi:10.1016/j.nic.2006.06.005

[19] Pierpaoli, C. and Basser, P.J. (1996) Toward a quantitative assessment of diffusion anisotropy. Magnetic Resonance in Medicine, 36, 893-906. doi: $10.1002 / \mathrm{mrm} .1910360612$

[20] Basser, P.J. (1995) Inferring microstructural features and the physiological state of tissues from diffusion-weighted images. NMR in Biomedicine, 8, 333-344. doi:10.1002/nbm.1940080707

[21] Murakami, R., Sugahara, T., Nakamura, H., et al. (2007) Malignant supratentorial astrocytoma treated with postoperative radiation therapy: Prognostic value of pretreatment quantitative diffusion-weighted MR imaging. Radiology, 243, 493-499. doi:10.1148/radiol.2432060450

[22] Field, A.S., Alexander, A.L., Wu, Y.C., et al. (2004) Diffusion tensor eigenvector directional color imaging patterns in the evaluation of cerebral white matter tracts altered by tumor. Journal of Magnetic Resonance Imaging, 20, 555-562. doi:10.1002/jmri.20169

[23] Mori, S., Frederiksen, K., van Zijl, P.C., et al. (2002) Brain white matter anatomy of tumor patients evaluated with diffusion tensor imaging. Annals of Neurology, 51, 377-380. doi:10.1002/ana.10137

[24] Clark, C.A., Barrick, T.R., Murphy, M.M. and Bell, B.A. (2003) White matter fiber tracking in patients with spaceoccupying lesions of the brain: A new technique for neurosurgical planning? NeuroImage, 20, 1601-1608. doi:10.1016/j.neuroimage.2003.07.022

[25] Coenen, V.A., Krings, T., Mayfrank, L., Polin, R.S., Reinges, M.H., Thron, A. and Gilsbach, J.M. (2001) Threedimensional visualization of the pyramidal tract in a neuronavigation system during brain tumor surgery: First experiences and technical note. Neurosurgery, 49, 86-93.

[26] Alexander, A.L., Badie, B. and Field, A.S. (2003) Diffusion tensor MRI depicts white matter reorganization after surgery. Proceedings of the ISMRM 11th Scientific Meeting, Berkeley, International Society of Magnetic Resonance in Medicine.

[27] Lu, S., Ahn, D., Johnson, G. and Cha, S. (2003) Peritumoral diffusion tensor imaging of high grade gliomas and metastatic brain tumors. American Journal of Neuroradiology, 24, 937-941.

[28] Yamada, K., Kizu, O., Mori, S., et al. (2003) Brain fiber tracking with clinically feasible diffusion-tensor MR imaging: Initial experience. Radiology, 227, 295-301. 
doi:10.1148/radiol.2271020313

[29] Tropine, A., Vucurevic, G., Delani, P., et al. (2004) Contribution of diffusion tensor imaging to delineation of gliomas and glioblastomas. Journal of Magnetic Resonance Imaging, 20, 905-912. doi:10.1002/jmri.20217

[30] Morita, K., Matsuzawa, H., Fujii, Y., Tanaka, R., Kwee, I.L. and Nakada, T. (2005) Diffusion tensor analysis of peritumoral edema using lambda chart analysis indicative of the heterogeneity of the microstructure within edema. Journal of Neurosurgery, 102, 336-341. doi:10.3171/jns.2005.102.2.0336

[31] Nimsky, C., Ganslandt, O. and Fahlbusch, R. (2005) Intraoperative diffusion-tensor MR imaging: Shifting of white matter tracts during neurosurgical procedures-Initial experience. Radiology, 234, 218-225. doi:10.1148/radiol.2341031984

[32] Nimsky, C., Gaslandt, O., Merfhor, D., et al. (2006) Intraoperative visualization of pyramidal tract by diffusiontensor-imaging-based fiber tracking. NeuroImage, 30, 12191229. doi:10.1016/j.neuroimage.2005.11.001

[33] Lu, S., Ahn, D., Johnson, G., Law, M., Zagzag, D. and Grossman, R.I. (2004) Diffusion-tensor MR imaging of intracranial neoplasia and associated peritumoral edema: Introduction of the tumor infiltration index. Radiology, 232, 221-228. doi:10.1148/radiol.2321030653

[34] Kamada, K., Houkin, K., Takeuchi, F., Ishii, N., Ikeda, J., Sawamura, Y., Kuriki, S., Kawaguchi, H. and Iwasaki, Y. (2003) Visualization of the eloquent motor system by integration of MEG, functional, and anisotropic diffusionweighted MRI in functional neuronavigation. Surgical Neurology, 59, 352-362. doi:10.1016/S0090-3019(03)00018-1

[35] Fan, G.G., Deng, Q.L., Wu, Z.H. and Guo, Q.Y. (2006) Usefulness of diffusion/perfusion-weighted MRI in patients with nonenhancing supratentorial brain gliomas: A valuable tool to predict tumour grading? British Journal of Radiology, 79, 652-658. doi:10.1259/bjr/25349497

[36] Law, M., Oh, S., Babb, J.S., et al. (2006) Low-grade gliomas: Dynamic susceptibility-weighted contrast-enhanced perfusion MR imaging: Prediction of patient clinical response. Radiology, 238, 658-667. doi:10.1148/radiol.2382042180

[37] Roberts, H.C., Roberts, T.P., Bollen, A.W., Ley, S., Brasch, R.C. and Dillon, W.P. (2001) Correlation of microvascular permeability derived from dynamic contrast-enhanced MR imaging with histologic grade and tumor labeling index: A study in human brain tumors. Academic Radiology, 8, 384-391. doi:10.1016/S1076-6332(03)80545-7

[38] Provenzale, J.M., York, G., Moya, M.G., Parks, L., Choma, M., Kealey, S., Cole, P. and Serajuddin, H. (2006) Correlation of relative permeability and relative cerebral blood volume in high-grade cerebral neoplasms. American Journal of Roentgenology, 187, 1036-1042. doi:10.2214/AJR.04.0676

[39] Covarubbias, D.J., Rosen, B.R. and Lev, M.H. (2004) Dynamic magnetic resonance perfusion imaging of brain tumors. The Oncologist, 9, 528-537. doi:10.1634/theoncologist.9-5-528
[40] Sibtain, N.A., Howe, F.A. and Saunders, D.E. (2007) The clinical value of proton magnetic resonance spectroscopy in adult brain tumours. Clinical Radiology, 62, 109-111. doi:10.1016/j.crad.2006.09.012

[41] Vuori, K., Kankaanranta, L., Häkkinen, A.M., Gaily, E., et al. (2004) Low-Grade gliomas and focal cortical developmental malformations: Differentiation with proton MR spectroscopy. Radiology, 230, 703-708. doi:10.1148/radiol.2303021804

[42] Fan, G., Sun, B., Wu, Z., Guo, Q. and Guo, Y. (2004) In vivo single-voxel proton MR spectroscopy in the differentiation of high-grade gliomas and solitary metastases. Clinical Radiology, 59, 77-85.

doi:10.1016/j.crad.2003.08.006

[43] Howe, F.A., Barton, S.J., Cudlip, S.A., et al. (2003) Metabolic profiles of human brain tumors using quantitative in vivo $1 \mathrm{H}$ magnetic resonance spectroscopy. Magnetic Resonance in Medicine, 49, 223-232. doi:10.1002/mrm.10367

[44] Law, M., Cha, S., Knopp, E.A., Johnson, G., Arnett, J. and Litt, A.W. (2002) High-grade gliomas and solitary metastases: Differentiation by using perfusion and proton spectroscopic MR imaging. Radiology, 222, 715-721. doi:10.1148/radiol.2223010558

[45] Moller-Hartmann, W., Herminghaus, S., Krings, T., et al. (2002) Clinical application of proton magnetic resonance spectroscopy in the diagnosis of intracranial mass lesions. Neuroradiology, 44, 371-381. doi:10.1007/s00234-001-0760-0

[46] Majos, C., Alonso, J., Aguilera, C., et al. (2003) Proton magnetic resonance spectroscopy ((1)H MRS) of human brain tumours: Assessment of differences between tumour types and its applicability in brain tumour categorization. European Radiology, 13, 582-591.

[47] Harting, I., Hartmann, M., Jost, G., et al. (2003) Differentiating primary central nervous system lymphoma from glioma in humans using localised proton magnetic resonance spectroscopy. Neuroscience Letters, 342, 163-166. doi:10.1016/S0304-3940(03)00272-6

[48] Castillo, M., Smith, J.K. and Kwock, L. (2000) Correlation of myo-inositol levels and grading of cerebral astrocytomas. American Journal of Neuroradiology, 21, 16451649.

[49] Galanaud, D., Nicoli, F., Chinot, O., et al. (2006) Noninvasive diagnostic assessment of brain tumors using combined in vivo MR imaging and spectroscopy. Magnetic Resonance in Medicine, 55, 1236-1245. doi:10.1002/mrm.20886

[50] Moller-Hartmann, W., Herminghaus, S., Krings, T., et al. (2002) Clinical application of proton magnetic resonance spectroscopy in the diagnosis of intracranial mass lesions. Neuroradiology, 44, 371-381. doi:10.1007/s00234-001-0760-0

[51] Stadlbauer, A., Gruber, S., Nimski, C., et al. (2006) Preoperative grading of gliomas using metabolite quantification with high-spatial-resolution proton MR spectroscopic imaging. Radiology, 238, 958-969. doi:10.1148/radiol.2382041896 


\section{ABBREVIATIONS}

ADC: Apparent Diffusion Coefficient

AF: Arcuate Fasciculus

ASL: Arterial Spin Labelling

BOLD: Blood Oxygen Level Dependent

CBF: Cerebral Blood Flow

Cr: Creatine

Cho: Choline

CSI: Chemical Shift Imaging

CST: Cortical Spinal tract

CT: Computed Tomography

DCS: Direct Cortical Stimulation

DNA: DeoxyriboNucleic Acid

DSC: Dynamic Susceptibility Contrast

DTI: Diffusion Tensor Imaging

DWI: Diffusion Weighted Imaging

FA: Fractional Anisotropy

EPI: Echo Planar imaging

fMRI: functional Magnetic Resonance Imaging

Gd: Gadolinium

Gd-DTPA: Gadolinium Diethylene Triamine Penta Acetate

Glu: Glutamate

Glx: Glutamine

${ }^{1}$ H-MRS: Hydrogen Magnetic Resonance Spectroscopy

HRF: Hemodynamic Response Function

IFOF: Inferior Fronto-Occipital Fasciculus
K2: First pass permaeability

Lac: Lactate

Lip: Lipids

LI: Lateralization Index

M1: Primarymotor cortex

MD: Mean Diffusivity

mI: myo Inositol

MR: Magnetic Resonance

MRI: Magnetic Resonance Imaging

MRS: Magnetic Resonance Spectroscopy

MTT: Mean Transit time

NAA: N-Acetyl Aspartate

NMR: Nuclear Magnetic Resonance

PWI: Perfusion Weighted Imaging

PET: Positron Emission Tomography

PCr: Phospho Creatine

RA: Relative Anisotropy

rCBV: Relative Cerebral Blood Flow

RF: Radiofrequency

ROI: Region of Interest

S1: Primary sensory cortex

SLF: Superior Longitudinal Fasciculus

SPECT: Single Photo Emission Tomography

SVS: Single Voxel Spectroscopy

TTP: Time To Peak

WHO: World Health Organization 